OPEN ACCESS

Edited by:

Martin Burtscher,

Universität Innsbruck, Austria

Reviewed by:

Amalia Peix,

Instituto de Cardiología y Cirugía

Cardiovascular, Cuba

Meijing Wang,

Indiana University Bloomington,

United States

${ }^{*}$ Correspondence:

Xiaohai Ma

maxi8238@yahoo.com

Mingwu Lou

mingwulou@sina.com

Liang Zhong

zhong.liang@nhcs.com.sg

Specialty section:

This article was submitted to Clinical and Translational Physiology,

a section of the journal

Frontiers in Physiology

Received: 11 May 2018

Accepted: 06 August 2018

Published: 03 September 2018

Citation:

Peng J, Zhao X, Zhao L, Fan Z, Wang Z, Chen H, Leng S, Allen J, Tan R-S, Koh AS, Ma X, Lou M and

Zhong L (2018) Normal Values of Myocardial Deformation Assessed by Cardiovascular Magnetic Resonance Feature Tracking in a Healthy Chinese

Population: A Multicenter Study.

Front. Physiol. 9:1181.

doi: 10.3389/fphys.2018.01181

\section{Normal Values of Myocardial Deformation Assessed by Cardiovascular Magnetic Resonance Feature Tracking in a Healthy Chinese Population: A Multicenter Study}

Junping Peng 1,2,3, Xiaodan Zhao ${ }^{4}$, Lei Zhao², Zhanming Fan², Zheng Wang ${ }^{2}$, Hui Chen², Shuang Leng ${ }^{4}$, John Allen ${ }^{5}$, Ru-San Tan ${ }^{4,5}$, Angela S. Koh ${ }^{4,5}$, Xiaohai Ma ${ }^{2 *}$, Mingwu Lou ${ }^{3 *}$ and Liang Zhong ${ }^{4,5 *}$

${ }^{1}$ Department of Radiology, The Eighth Affiliated Hospital, Sun Yat-sen University, Shenzhen, China, ${ }^{2}$ Department of Radiology, Beijing Anzhen Hospital, Capital Medical University, Beijing, China, ${ }^{3}$ Post-Doctoral Research Center, Department of Radiology, Longgang Central Hospital, Shenzhen Clinical Medical Institute, Guangzhou University of Chinese Medicine, Shenzhen, China, ${ }^{4}$ National Heart Centre Singapore, Singapore, Singapore, ${ }^{5}$ Duke-NUS Medical School, Singapore, Singapore

Reference values on atrial and ventricular strain from cardiovascular magnetic resonance $(\mathrm{CMR})$ are essential in identifying patients with impaired atrial and ventricular function. However, reference values have not been established for Chinese subjects. One hundred and fifty healthy volunteers (75 Males/75 Females; 18-82 years) were recruited. All underwent CMR scans with images acceptable for further strain analysis. Subjects were stratified by age: Group 1, 18-44 years; Group 2, 45-59 years; Group 3, $\geq 60$ years. Feature tracking of CMR cine imaging was used to obtain left atrial global longitudinal $\left(\left\llcorner A E_{\|}\right)\right.$and circumferential strains ( $\left.L A E_{C C}\right)$ and respective systolic strain rates, left ventricular longitudinal (LV $\left.E_{\|}\right)$, circumferential (LV $\left.E_{\mathrm{CC}}\right)$ and radial strains (LV $\left.E_{r r}\right)$ and their respective strain rates, and right ventricular longitudinal strain (RV $\left.E_{\|}\right)$and strain rate. $L A E_{\|}$and $L A E_{\mathrm{CC}}$ were $32.8 \pm 9.2 \%$ and $40.3 \pm 13.4 \%$, respectively, and $R V$ $E_{\|}$was $-29.3 \pm 6.0 \%$. LV $E_{\|}, \mathrm{LV} E_{\mathrm{cc}}$ and LV $E_{\mathrm{rr}}$ were $-22.4 \pm 2.9 \%,-24.3 \pm 3.1 \%$ and $79.0 \pm 19.4 \%$, respectively. LV $E_{\|}$and LV $E_{C C}$ were higher in females than males $(P<0.05)$. LA $E_{\|}, L A E_{\mathrm{CC}}$, and LV $E_{\mathrm{Cc}}$ decreased, while LV $E_{\mathrm{rr}}$ increased with age $(P<0.05)$. LV $E_{\|}$and $R V E_{\|}$were not shown to be associated with age. Normal ranges for atrial and ventricular strain and strain rates are provided using CMR feature tracking in Chinese subjects.

Keywords: cardiovascular magnetic resonance, feature tracking, strain and deformation, Chinese, multicenter

\section{INTRODUCTION}

Assessment of chamber function is an important objective of a cardiac imaging study. In assessing chamber function, myocardial deformation is superior to left ventricular (LV) ejection fraction for prognostication in patients with various myocardial disorders (Stanton et al., 2009; Motoki et al., 2012; Zhong et al., 2013; Kalam et al., 2014). Several advanced techniques based on either echocardiography or cardiovascular magnetic resonance (CMR), 
such as real-time speckle-tracking echocardiography (Zhang et al., 2016), tissue tagging and feature tracking (Zhong et al., 2012; Venkatesh et al., 2014; Khan et al., 2015), have become available for assessing myocardial deformation. Advantages of these techniques include excellent scan-rescan reproducibility, less dependence on operator technique, and more accurate and reproducible measures of the left and right ventricles. Consequently, CMR has become the standard reference modality for measurement of ventricular volume and function (Bellenger et al., 2000), and arguably the optimal imaging modality for quantification of myocardial displacement (Leng et al., 2015, 2016, 2018), strain and strain rate (Rahman et al., 2017; Scatteia et al., 2017; Koh et al., 2018; Zhao et al., 2018).

Cardiovascular magnetic resonance feature tracking (CMR-FT) is analogous to speckle-tracking echocardiography and allows quantification of global and regional myocardial motion and deformation using standard, balanced steady state free precession (SSFP)/balanced turbo field echo (BTFE) cine $\mathrm{CMR}$ images in long and short axis views, which are imperative in routine clinical CMR practice (Augustine et al., 2013). Reference values of cardiac strain for the Western population have been reported in Andre et al. (2015), Kawel-Boehm et al. (2015), Taylor et al. (2015), Mangion et al. (2016). Distribution patterns of LV myocardial strain in healthy Chinese volunteers were provided using deformation registration algorithm (TrufiStrain, Siemens Healthcare), however, strain analysis for left atrium and right ventricle was not performed (Liu et al., 2017).

In this study, we aimed to establish CMR reference values for left atrial (LA), left ventricular (LV), and right ventricular (RV) strains and strain rate in Chinese subjects, and to evaluate the effects of age and gender on strain and strain rate measurements.

\section{MATERIALS AND METHODS}

\section{Population}

Healthy volunteers who met the following inclusion criteria were prospectively recruited from two centers in China
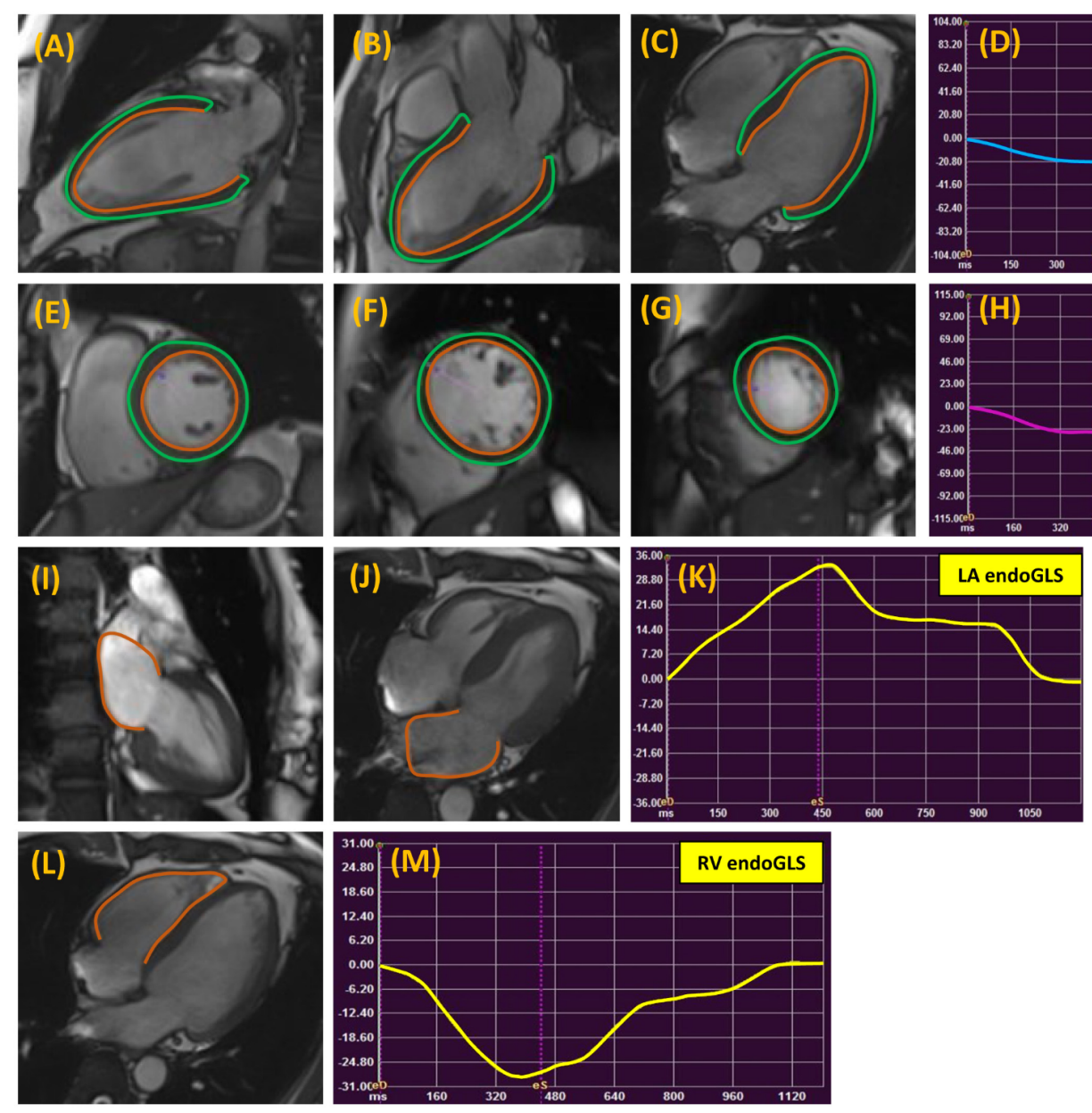

FIGURE 1 | Representative images in the standard long axis (A-C) and short axis (E-G) orientations and strain curves. Contours are illustrated in left ventricular endocardial and epicardial borders in the 2-, 3-, 4-chamber (A-C) and corresponding LV MyoGLS (LV E॥) (D); endocardial and epicardial borders in base, mid, apical short axis views (E-G) and corresponding LV MyoGCS (LV $\left.E_{\mathrm{CC}}\right)(\mathbf{H})$; left atrial endocardial borders in 2-, and 4-chamber (I,J) and corresponding LA endoGLS (LA E॥) (K); right ventricular endocardial border in 4-chamber (L) and corresponding RV endoGLS (RV E $E_{\|}$(M). 


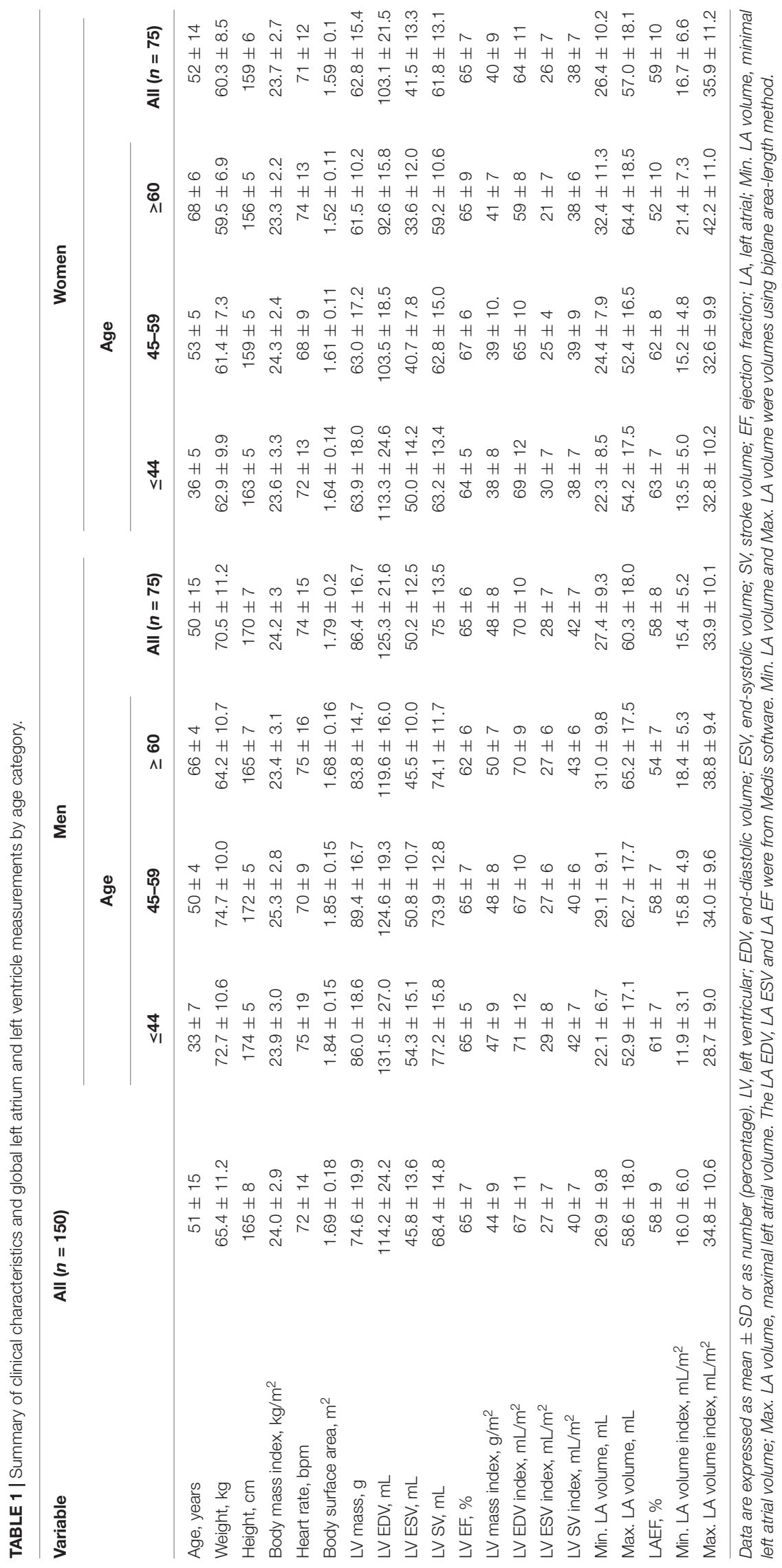



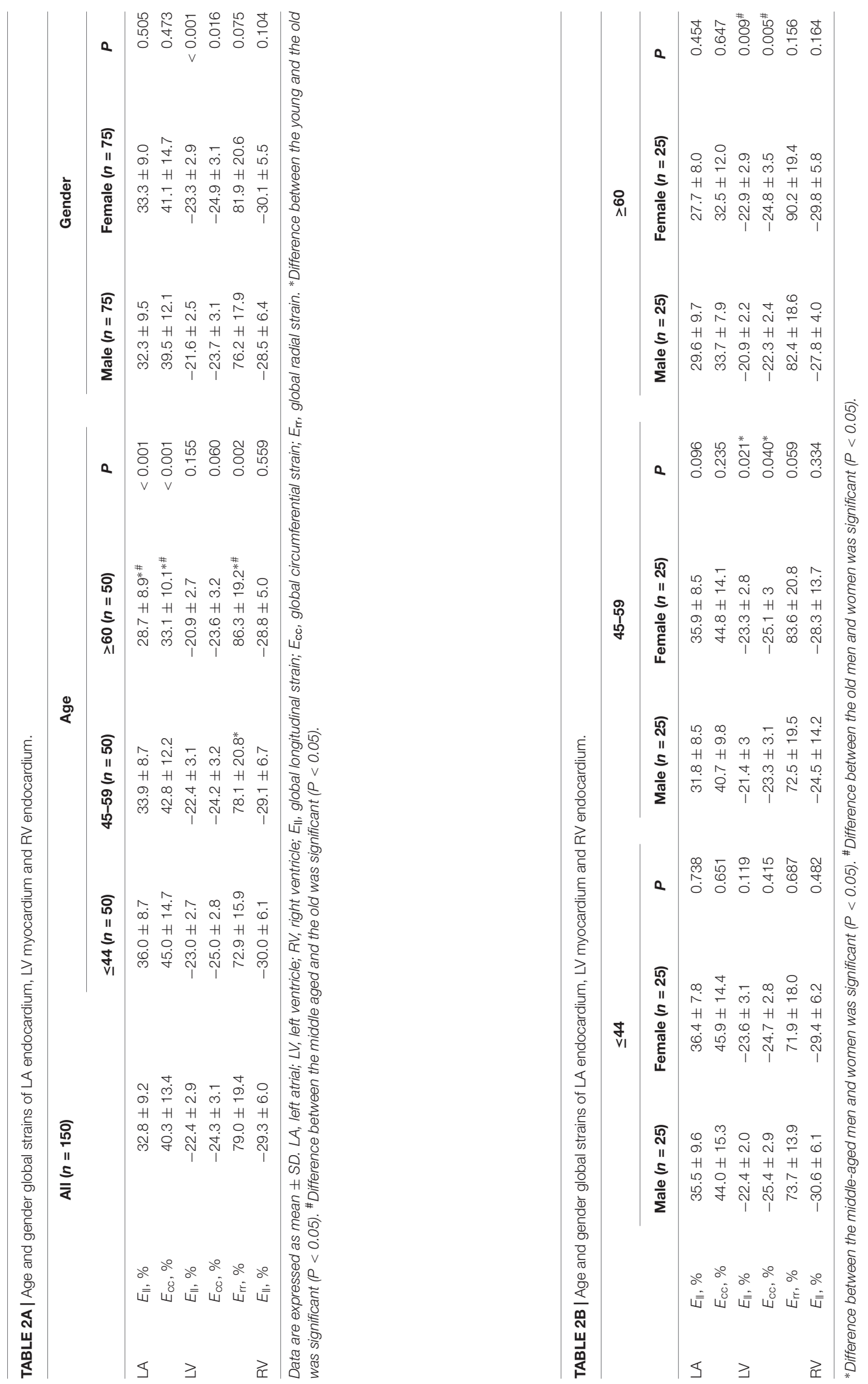
and one in Singapore: (1) no symptoms or prior history of cardiovascular or cerebrovascular disease; (2) no prior diagnosis of hyperlipidaemia, hypertension or diabetes mellitus; (3) normal physical examination and electrocardiogram; (4) no contraindications to CMR. Subjects with wall motion abnormalities or significant valvular diseases detected on CMR were excluded. The study was approved by the local institutional research ethics committee. Written informed consent was obtained from all subjects. The study protocol conforms to the ethical guidelines of the 1975 Declaration of Helsinki as reflected in a priori approval by the institution's human research committee.

\section{CMR Image Acquisition}

Subjects underwent cine CMR on a $1.5 \mathrm{~T}$ (Verio, Siemens, Germany) or a 3.0T (Prisma, Siemens, Germany) CMR scanners at the China sites and on a 3.0T (3.0T, Ingenia, Philips Healthcare, Netherlands) CMR scanner at the Singapore site, using a standardized imaging protocol (Kramer et al., 2013). Balanced SSFP (China sites) and BTFE (Singapore site) sequences with breath-hold were performed to obtain cine CMR images, comprising a stack of contiguous parallel short-axis slices covering the entire LV and RV from base to apex and three LV long-axis slice (2-, 3-, and 4-chamber views) images. The slice thickness/spacing is $5 \mathrm{~mm} / 1 \mathrm{~mm}$ for long axis and $8 \mathrm{~mm} / 2 \mathrm{~mm}$ for short axis in China sites; and $8 \mathrm{~mm} / 0 \mathrm{~mm}$ for both short and long axis in Singapore site.

\section{CMR Image Post-processing}

Cine CMR image manual segmentation and analyses were conducted by investigators experienced in CMR (JPP, XDZ, and ZW). Minimal and maximal left atrial volumes were calculated at the respective cardiac phases using biplane area-length method (Lang et al., 2005): LA volume $(\mathrm{mL})=0.85^{*} \mathrm{~A} 2 \mathrm{C}^{*} \mathrm{~A} 4 \mathrm{C} / \mathrm{L}$, where $\mathrm{A} 2 \mathrm{C}$ and $\mathrm{A} 4 \mathrm{C}$ represent planimetered LA area in the 2- and 4-chamber views, respectively; and L, the length of the major LA axis at either the 2- or 4-chamber view, whichever is shorter. LV end-diastolic volume (LVEDV), end-systolic volume (LVESV), stroke volume (LVSV), ejection fraction (LVEF) and LV mass were measured using Qmass (Medis Suite, Netherlands), and applicable values indexed to body surface area were calculated.

\section{CMR Feature Tracking Analysis}

Global and regional LA, LV, and RV strain and systolic strain rate measurements were analyzed using commercial cardiovascular post-processing software (Medis 3.0, Netherlands). Feature tracking allows quantification of global and regional longitudinal, circumferential and radial strain and strain rates. A short description of the analysis is given here. At end-diastole, endocardial and epicardial borders were manually delineated using a point-and-click approach before the automated tracking algorithm was applied. Papillary muscles included within the endocardial borders (Figures 1A-C,E-G). LA endocardial contours were initially traced in the 2- and 4- chamber views at the minimum LA volume after atrial contraction (Figures 1I,J). RV endocardial border was traced in 4-chamber view (Figure 1L). Guided by signal in homogeneities or anatomical features and using a maximum likelihood method, the software algorithm provides an automatically traced image with frame-to-frame template matching throughout the entire cardiac cycle. Strain values are derived by comparing the movement of the features in relation to each other along the initially drawn borders. CMR feature tracking performance was visually reviewed to ensure accurate tracking. In cases where tracking is determined to be inadequate, the software allows for border editing. Global longitudinal, circumferential and radial strain values $\left(E_{\mathrm{ll}}, E_{\mathrm{cc}}\right.$, and $\left.E_{\mathrm{rr}}\right)$ were automatically extracted from corresponding strain curves (Figures 1D,H,K,M).

LA longitudinal strain and strain rate were measured in both 2- and 4-chamber views, excluding the confluence of the pulmonary veins and LA appendage in the border delineation. The LA wall contours were automatically segmented into anterior, inferior, posterior, lateral and atrial septal regions by the software (Kowallick et al., 2014; Zareian et al., 2015).

The standard 17-segment model of the American Heart Association (AHA) (Cerqueira et al., 2002) was applied in the analysis of LV longitudinal strain measured in 2-, 3-, and 4-chamber views. The basal, mid-cavity and apical levels were segmented from the end-diastolic 4-chamber long-axis cine image. For circumferential and radial strain analyses of LV short-axis views, a modified 16-segment model (omitting the apical cap) was applied, using the RV insertion point as the reference point for the junction of LV anterior wall and septum. For short axis strain analysis, base was selected as the slice still showing a complete circumference of myocardium throughout the entire cardiac cycle (without through plane distortion from the LV outflow tract) and the apex was selected as the slice still showing LV cavity at systole (Taylor et al., 2015; Kowallick et al., 2016).

Segmental endocardial and myocardial values of LV were separately measured for longitudinal and circumferential strain and strain rates. To dissect the regional strain distribution, we calculated longitudinal and circumferential strains at basal, middle and apical levels, as well as at the anterior wall, septal, inferior wall and lateral walls of LV by averaging the peak values of the segments corresponding to the relevant territories. For each segment, strain rate was calculated as the derivative of the initial measured strain.

Global LA longitudinal strain (and strain rate) was calculated as the average of strain (and strain rate) from 2-and 4-chamber views, and regional segments were divided as anterior wall, inferior wall, roof, lateral wall and septum wall. The values at roof was taken as the average value from 2- and 4-chamber views. Global and regional RV (free wall and septum) longitudinal strains were measured on the 4-chamber view, and the respective strain rates were calculated (Heermann et al., 2014; Prati et al., 2015).

\section{Statistical Analysis}

All continuous variables were described as mean \pm standard deviation (SD). Means of baseline variables among the three age groups were compared using one-way analysis of variance (ANOVA) with post hoc pairwise comparisons and the Bonferroni correction for multiple comparisons; 
TABLE 3 | Left ventricular segmental endocardial and myocardial circumferential strain and strain rates from short axis view.

\begin{tabular}{lcccc}
\hline Segment & $\begin{array}{c}\text { Circumferential } \\
\text { endocardial strain, } \%\end{array}$ & $\begin{array}{c}\text { Circumferential endocardial } \\
\text { strain rate, s } \mathbf{~}^{\mathbf{1}}\end{array}$ & $\begin{array}{c}\text { Circumferential } \\
\text { myocardial strain, \% }\end{array}$ & $\begin{array}{c}\text { Circumferential myocardial } \\
\text { strain rate, s }\end{array}$ \\
\hline 1 & $-33.2 \pm 8.9$ & $-1.80 \pm 0.55$ & $-21.1 \pm 6.8$ & $-1.12 \pm 0.37$ \\
2 & $-33.9 \pm 8.5$ & $-1.87 \pm 0.61$ & $-20.7 \pm 6.4$ & $-1.07 \pm 0.37$ \\
3 & $-31.1 \pm 8.4$ & $-1.63 \pm 0.55$ & $-23.6 \pm 6.1$ & $-1.16 \pm 0.38$ \\
4 & $-31.4 \pm 7.6$ & $-1.58 \pm 0.49$ & $-20.8 \pm 6.5$ & $-0.94 \pm 0.33$ \\
5 & $-37.3 \pm 6.3$ & $-2.07 \pm 0.55$ & $-28.8 \pm 6.5$ & $-1.46 \pm 0.41$ \\
6 & $-38.6 \pm 7.2$ & $-2.08 \pm 0.58$ & $-31.3 \pm 7.3$ & $-1.61 \pm 0.50$ \\
7 & $-34.0 \pm 8.6$ & $-1.92 \pm 0.63$ & $-18.4 \pm 6.6$ & $-1.01 \pm 0.35$ \\
8 & $-34.6 \pm 9.5$ & $-1.96 \pm 0.71$ & $-19.7 \pm 6.5$ & $-1.07 \pm 0.43$ \\
9 & $-32.3 \pm 8.6$ & $-1.75 \pm 0.58$ & $-23.7 \pm 5.4$ & $-1.18 \pm 0.33$ \\
10 & $-31.8 \pm 7.2$ & $-1.66 \pm 0.46$ & $-21.5 \pm 5.8$ & $-1.07 \pm 0.32$ \\
11 & $-36.0 \pm 6.4$ & $-1.98 \pm 0.57$ & $-25.5 \pm 6.2$ & $-1.36 \pm 0.46$ \\
12 & $-29.6 \pm 9.0$ & $-1.60 \pm 0.58$ & $-21.8 \pm 8.0$ & $-1.25 \pm 0.43$ \\
13 & $-41.3 \pm 9.2$ & $-2.34 \pm 0.79$ & $-27.6 \pm 7.1$ & $-1.4 \pm \pm 0.48$ \\
15 & $-44.1 \pm 9.6$ & $-2.48 \pm 0.84$ & $-32.1 \pm 7.2$ & $-1.65 \pm 0.53$ \\
16 & $-45.3 \pm 9.9$ & $-2.53 \pm 0.86$ & $-29.1 \pm 7.6$ & $-1.63 \pm 0.52$ \\
\hline
\end{tabular}

Data are expressed as mean $\pm S D$. 1, basal anterior; 2, basal anterolateral; 3, basal inferoseptal; 4, basal inferior; 5, basal inferolateral; 6, basal anterolateral; 7, mid anterior; 8, mid anteroseptal; 9, mid inferoseptal; 10, mid inferior; 11, mid inferolateral; 12, mid anterolateral; 13, apical anterior; 14, apical septal; 15, apical inferior; 16, apical lateral.

comparisons between males and females were performed using a two-sample $t$-test. Correlation was assessed using the Pearson correlation coefficient $(r)$. Statistical significance was set at $P \leq 0.05$. All analyses were performed using SPSS Statistics 22.0. Intra- and inter-observer reproducibility were performed in 20 randomly selected subjects using intra-class coefficient (ICC), Bland-Altman method and the coefficient of variation $(\mathrm{CV})$, which was calculated as the mean of absolute difference between two methods over the mean average.

\section{RESULTS}

\section{Clinical Characteristics of Study Subjects}

One hundred and fifty subjects (75 Males/75 Females, 18-82 years) with cine CMR images acceptable for CMR feature tracking analysis were recruited. A summary of clinical characteristics and global left atrium and left ventricle measurements by age category are presented in Table 1. Of the 150 subjects, 84 were from Beijing Anzhen Hospital [40 males, mean $( \pm \mathrm{SD})$ age $43 \pm 12$ years], 41 from National Heart Centre Singapore (24 males, mean age $66 \pm 8$ years), and 25 from Longgang Central Hospital of Shenzhen (12 males; mean age $54 \pm 11$ years $)$.

\section{Global and Regional Strain Values}

Normal values for global LA endocardial, LV myocardial and RV endocardial CMR feature-tracking deformation measured parameters are shown in Table 2A. Mean endocardial LA $E_{11}$ and LA $E_{\mathrm{cc}}$ were $32.8 \pm 9.2$ and $40.3 \pm 13.4 \%$, respectively. For LA $E_{1 l}$ at the segmental level, the LA roof exhibited the lowest strain
(30.7 $\pm 13.9 \%$ ), and differences between the LA roof and LA $E_{11}$ at the anterior $(38.7 \pm 18.5 \%)$ and inferior $(41.3 \pm 20.4 \%)$ walls were both significant $(P \leq 0.003)$. LA strain rate at the anterior wall $\left(1.66 \pm 0.89 \mathrm{~s}^{-1}\right)$ was significantly higher than at the LA roof $\left(1.35 \pm 0.52 \mathrm{~s}^{-1}\right)$, lateral wall $\left(1.30 \pm 0.63 \mathrm{~s}^{-1}\right)$ and septal wall $\left(1.40 \pm 0.61 \mathrm{~s}^{-1}\right)($ all $P \leq 0.01)$. Age and gender specific global LA endocardial, LV myocardial and RV endocardial strain values are given in Table $\mathbf{2 B}$.

For LV myocardium strains, mean LV $E_{11}, \mathrm{LV} E_{\mathrm{cc}}$ and $\mathrm{LV} E_{\mathrm{rr}}$ was $-22.4 \pm 2.9,-24.3 \pm 3.1$, and $79.0 \pm 19.4 \%$, respectively. The endocardial and myocardial circumferential strain and strain rates for regional 16 segments are provided in Table 3. Barchart plots (with one standard deviation) for segmental LV endocardial and myocardial circumferential strains and strain rates at basal, mid-cavity and apical levels, and at the anterior, septum, inferior and lateral walls are given in Figures 2A-D. Means for both endocardial and myocardial LV $E_{\mathrm{cc}}$ were significantly higher at the apical level than at the base and mid-cavity levels (all $P<0.001$ ), and the mid-level exhibited the lowest strain among the three levels $(P<0.001)$. For both endocardial and myocardial circumferential strain rates, significantly larger values were observed at the apical level compared to mid-cavity $(P<0.001)$. Furthermore, myocardial $E_{\mathrm{cc}}$ and strain rate increased significantly from anterior $\rightarrow$ septum $\rightarrow$ inferior $\rightarrow$ lateral walls (all $P<0.001$ ), while no differences were found for endocardial $E_{\mathrm{cc}}$ and strain rate among these four regional walls. In contrast, endocardial LV $E_{1 l}$ increased significantly from basal to mid to apex (Figure 2E), and mean myocardial LV $E_{\mathrm{ll}}$ was higher at the middle level rather than at the basal and apical levels (Figure 2G). Lateral walls had significantly higher endocardial and myocardial $E_{11}$ and strain rates than anterior walls, and inferior and septum walls, with septum walls having the lowest strain and strain rates 

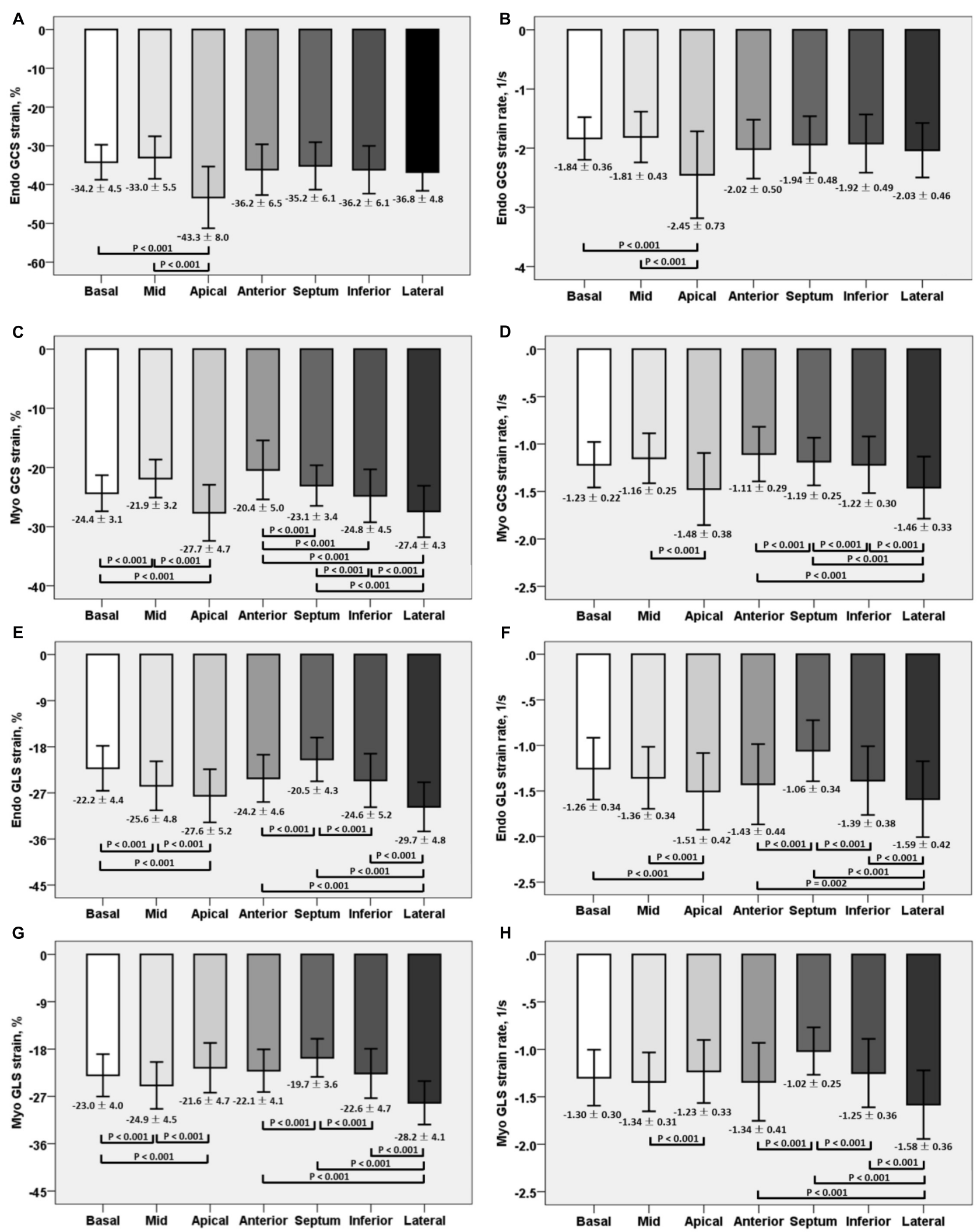

FIGURE 2 | Comparison of endocardial circumferential strain and strain rate (A) and (B); myocardial circumferential strain and strain rate (C) and (D); endocardial longitudinal strain and strain rate (E) and (F); and myocardial longitudinal strain and strain rate (G) and (H) at basal, mid and apical levels; anterior, septum, inferior and lateral walls. 
TABLE 4 | Left ventricular segmental endocardial and myocardial longitudinal strain and strain rates from long axis view.

\begin{tabular}{lcccc}
\hline Segment & $\begin{array}{c}\text { Longitudinal } \\
\text { endocardial strain, } \%\end{array}$ & $\begin{array}{c}\text { Longitudinal endocardial } \\
\text { strain rate, } \mathbf{s}^{-\mathbf{1}}\end{array}$ & $\begin{array}{c}\text { Longitudinal } \\
\text { myocardial strain, \% }\end{array}$ & $\begin{array}{c}\text { Longitudinal myocardial } \\
\text { strain rate, } \mathbf{s}^{-1}\end{array}$ \\
\hline 1 & $-14.8 \pm 7.8$ & $-1.01 \pm 0.58$ & $-12.6 \pm 7.5$ & $-0.98 \pm 0.59$ \\
2 & $-15.3 \pm 9.1$ & $-0.93 \pm 0.46$ & $-19.0 \pm 8.1$ & $-1.03 \pm 0.44$ \\
3 & $-18.8 \pm 6.9$ & $-0.88 \pm 0.55$ & $-18.1 \pm 6.3$ & $-0.88 \pm 0.32$ \\
4 & $-20.1 \pm 8.8$ & $-1.29 \pm 0.60$ & $-22.1 \pm 8.8$ & $-1.30 \pm 0.60$ \\
5 & $-32.5 \pm 8.0$ & $-1.79 \pm 0.53$ & $-33.4 \pm 8.8$ & $-1.84 \pm 0.58$ \\
6 & $-31.7 \pm 8.6$ & $-1.64 \pm 0.68$ & $-32.9 \pm 8.8$ & $-1.77 \pm 0.59$ \\
7 & $-23.9 \pm 10.8$ & $-1.41 \pm 0.63$ & $-23.1 \pm 10.8$ & $-1.35 \pm 0.61$ \\
8 & $-22.9 \pm 7.9$ & $-1.22 \pm 0.47$ & $-20.7 \pm 7.8$ & $-1.10 \pm 0.44$ \\
9 & $-23.7 \pm 8.0$ & $-1.11 \pm 0.59$ & $-24.0 \pm 7.4$ & $-1.17 \pm 0.44$ \\
10 & $-22.5 \pm 9.8$ & $-1.20 \pm 0.49$ & $-23.5 \pm 9.4$ & $-1.23 \pm 0.51$ \\
11 & $-31.0 \pm 8.8$ & $-1.62 \pm 0.60$ & $-28.5 \pm 10.5$ & $-1.62 \pm 0.65$ \\
13 & $-29.8 \pm 9.8$ & $-1.57 \pm 0.73$ & $-30.7 \pm 9.9$ & $-1.59 \pm 0.67$ \\
14 & $-33.9 \pm 8.9$ & $-1.86 \pm 0.69$ & $-16.6 \pm 5.7$ & $-0.91 \pm 0.32$ \\
15 & $-21.7 \pm 6.7$ & $-1.16 \pm 0.42$ & $-22.3 \pm 10.7$ & $-1.22 \pm 0.53$ \\
17 & $-31.1 \pm 9.6$ & $-1.67 \pm 0.64$ & $-16.6 \pm 7.9$ & $-1.10 \pm 0.43$ \\
\hline
\end{tabular}

Data are expressed as mean $\pm S D .1$, basal anterior; 2, basal anterolateral; 3, basal inferoseptal; 4, basal inferior; 5, basal inferolateral; 6, basal anterolateral; 7, mid anterior; 8, mid anteroseptal; 9, mid inferoseptal; 10, mid inferior; 11, mid inferolateral; 12, mid anterolateral; 13, apical anterior; 14, apical septal; 15, apical inferior; 16, apical lateral; 17, apex.

(Figures 2F,H). Endocardial and myocardial longitudinal strain and strain rates for the regional 17 segments are provided in Table 4.

Mean RV $E_{11}$ was $-29.3 \pm 6.0 \%$, with RV free wall exhibiting greater longitudinal strain $(-35.3 \pm 7.4 \%$ vs. $-23.9 \pm 6.9 \%)$ and strain rate $\left(-1.83 \pm 0.46 \mathrm{~s}^{-1}\right.$ vs. $\left.-1.12 \pm 0.39 \mathrm{~s}^{-1}\right)$ than the septum (both $P<0.001$ ).

\section{Age and Gender Difference in Global and Regional Strain and Strain Rate}

Global strain values for LA, LV, and RV among age groups is given in Table 2. For age groups 1, 2, and 3, respectively, global strain values were $36.0 \pm 8.7,33.9 \pm 8.7$, and $28.7 \pm 8.9 \%$ for LA $E_{\mathrm{ll}}$, and $45.0 \pm 14.7,42.8 \pm 12.2$, and $33.1 \pm 10.1 \%$ for LA $E_{\mathrm{cc}}$. LA $E_{\mathrm{ll}}$ and $E_{\mathrm{cc}}$ decreased in magnitude with increasing age; correlations were statistically significant but weak ( $r=-0.330$ for LA $E_{\mathrm{ll}}$ and $r=-0.364$ for LA $\left.E_{\mathrm{cc}}\right)$. LA endocardial strain differences between male and female were non-significant, with values of $32.3 \pm 9.5 \%$ vs. $33.3 \pm 8.9 \%$ for $E_{11}(P=0.505)$ and $39.5 \pm 12.1 \%$ vs. $41.1 \pm 14.7 \%$ for $E_{\mathrm{cc}}$ $(P=0.473)$.

In age groups 1,2 , and 3 , mean values for $\mathrm{LV}$ myocardial $E_{11}$ were $-23.0 \pm 2.7,-22.4 \pm 3.1$, and $-20.9 \pm 2.7$; for $\mathrm{LV}$ $E_{\mathrm{cc}}-25.0 \pm 2.8,-24.2 \pm 3.2$, and $-23.6 \pm 3.2 \%$; and for $\mathrm{LV} E_{\mathrm{rr}} 72.8 \pm 15.9,78.1 \pm 20.8$, and $86.3 \pm 19.2 \%$. Linear regression showed weak but statistically significant associations, respectively, of $E_{\mathrm{cc}}$ and $E_{\mathrm{rr}}$ with age $(r=0.208$ and $r=0.258$, both $P<0.05)$. Differences between males and females were significant for LV $E_{11}(-21.6 \pm 2.5 \%$ vs. $-23.3 \pm 2.9 \%)$ and LV $E_{\mathrm{cc}}(-23.7 \pm 3.1 \%$ vs. $-24.9 \pm 3.1 \%)$ (both $\left.P<0.05\right)$. Difference in $\mathrm{LV} E_{\mathrm{rr}}$ between genders $(76.2 \pm 17.9 \%$ vs. $81.9 \pm 20.6 \%)$ was non-significant $(P=0.075)$. Comparison of male and female for three age groups with $P$-value was tabulated in Table 2B. Age and gender specific endocardial and myocardial $E_{\mathrm{cc}}$ values for 16 segments are given in Tables 5, 6, and endocardial and myocardial $E_{11}$ for 17 segments are tabulated in Tables 7, 8.

RV $E_{11}$ showed no significant differences among three age groups $(-30.0 \pm 6.1,-29.1 \pm 6.7$, and $-28.8 \pm 5.0 \%$, $P=0.559) . E_{11}$ values in males and females were $-28.5 \pm 6.4$ and $-30.1 \pm 5.5 \%$, but not statistically significant $(P=0.104)$.

\section{Reproducibility}

The intra- and inter-observer variability results were given in Table 9. In Bland-Altman analyses, LV $E_{\mathrm{cc}}$ had the best intraobserver agreement (bias, $-0.04 \pm 0.72 ; 95 \% \mathrm{CI},-1.48$ to 1.41 ), and $L V E_{l l}$ had the best inter-observer agreement (bias, $-0.07 \pm 0.77 ; 95 \% \mathrm{CI},-1.61$ to 1.47$)$. All parameters had an intra- and inter- observer ICC $>0.89$, except for LV $E_{\mathrm{rr}}$ with intra- (0.793) and inter- (0.832) observer.

\section{DISCUSSION}

To the best of our knowledge, this is the largest prospective study to date that quantifies global, segmental and regional strain and strain rates of healthy Chinese across a broad age range. Based on results obtained using the CMR feature tracking technique, our study demonstrates (i) higher magnitudes of longitudinal and circumferential LA and LV strain in females; (ii) regional variations in longitudinal and circumferential strains, with higher strain values in the lateral LV territories and RV free wall compared to the septal area; and (iii) independent associations between age and LA and LV global circumferential 


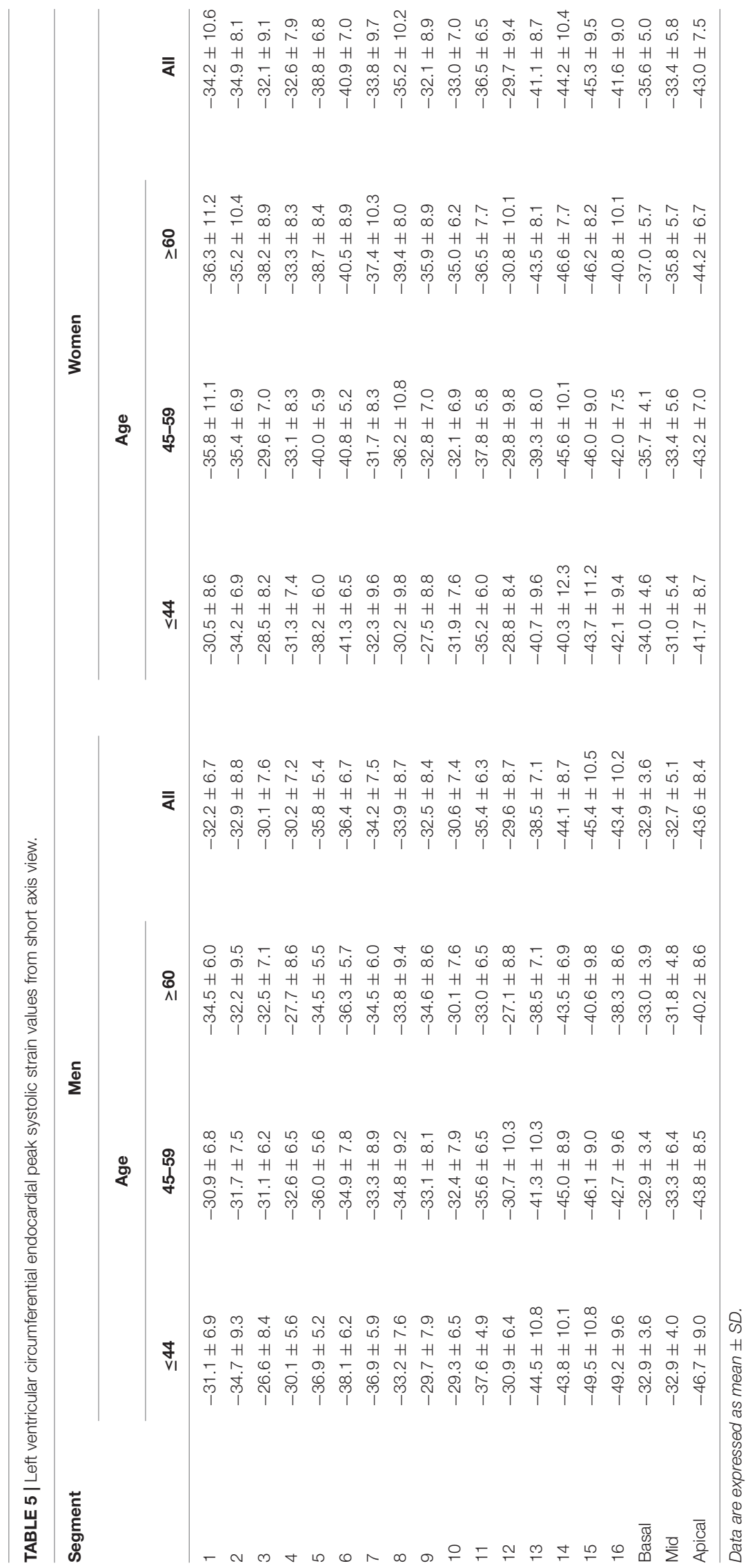




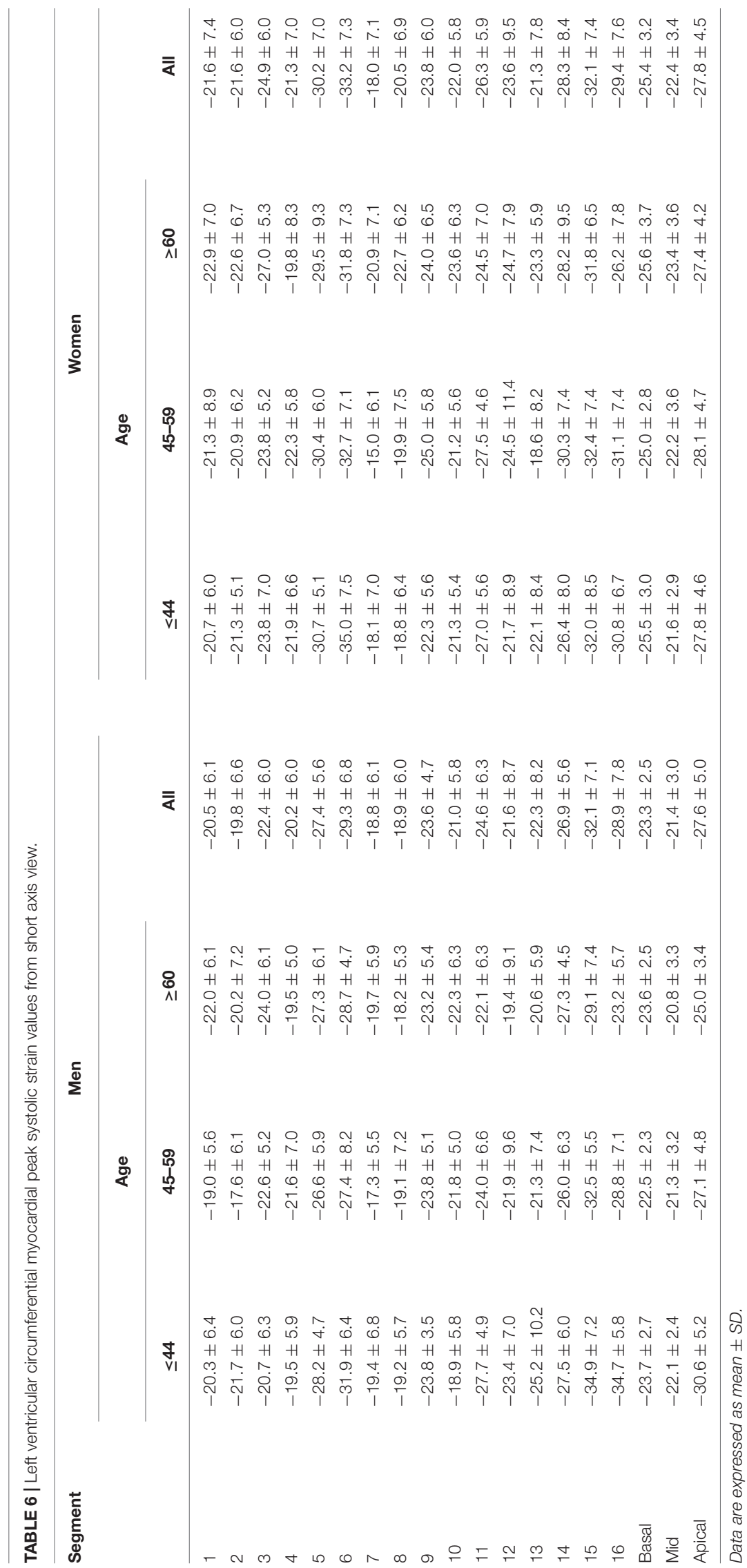




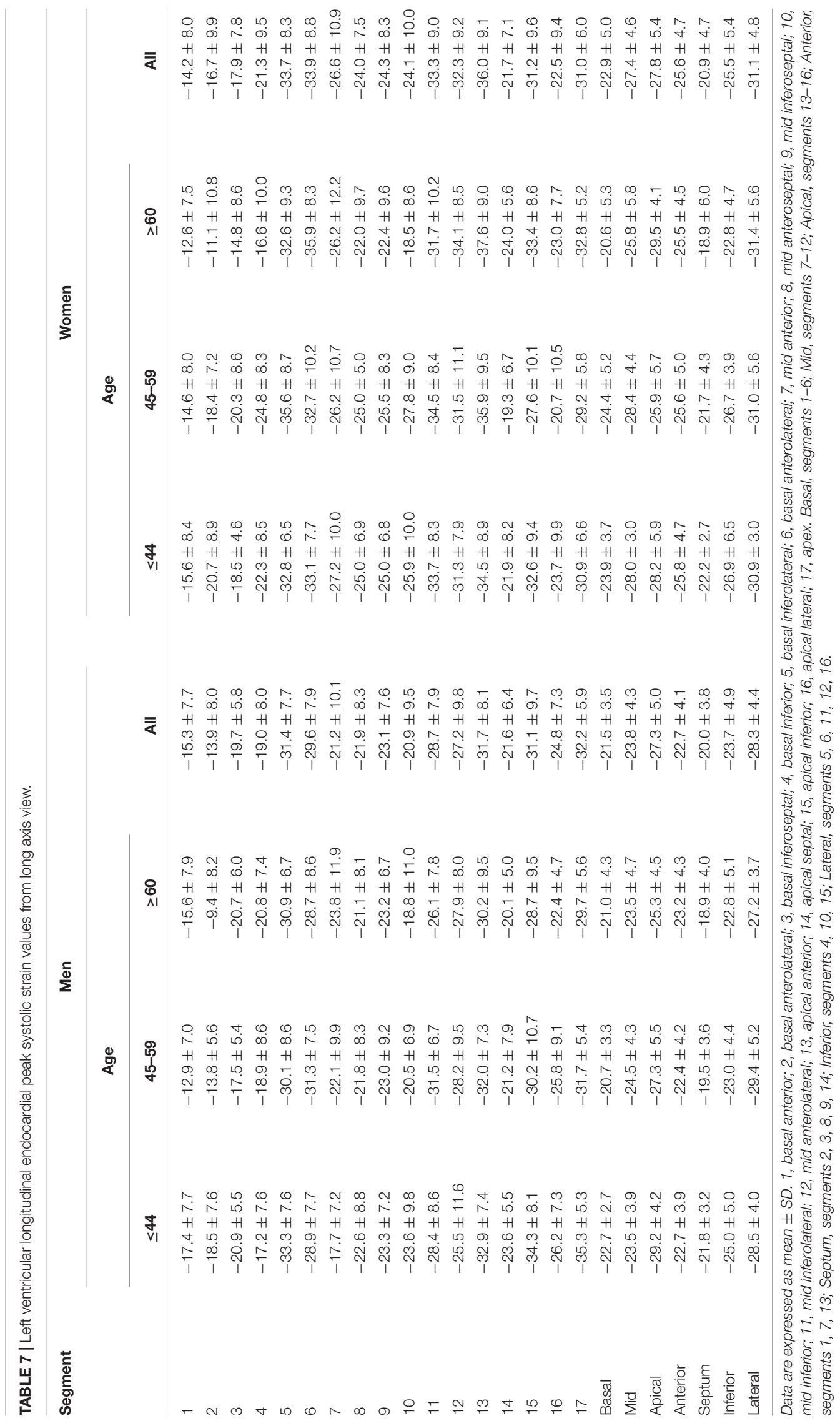




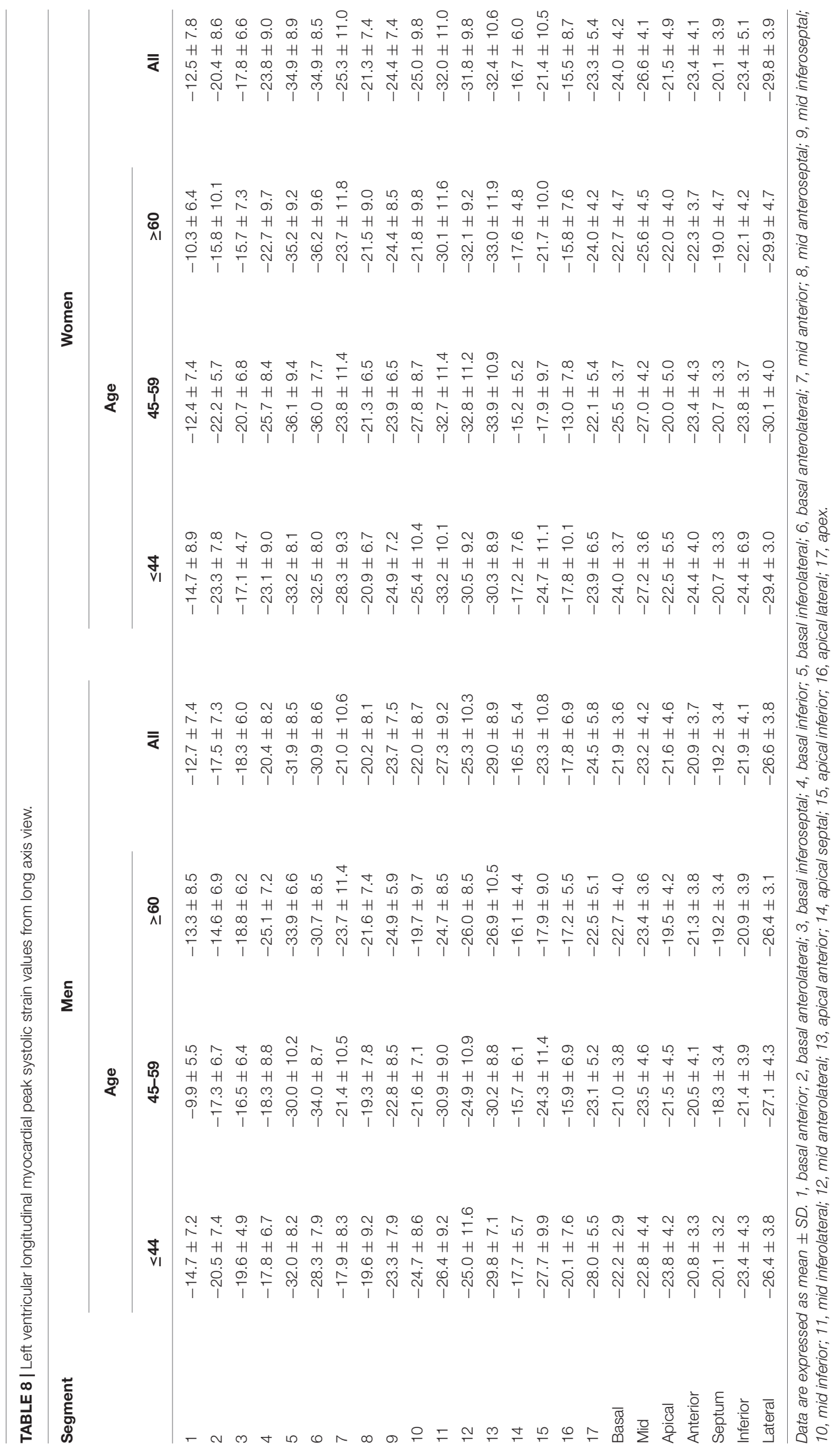


TABLE 9 | Intra-observer and inter-observer variability.

\begin{tabular}{|c|c|c|c|c|c|}
\hline Variable & Variability & Mean bias \pm SD & Limits of agreement & Coefficient of variation (\%) & ICC (95\% Cl) \\
\hline \multirow[t]{2}{*}{$\left\llcorner A E_{\|}\right.$} & Intra-observer & $-0.41 \pm 1.24$ & -2.88 to 2.07 & 2.43 & $0.994(0.985,0.998)$ \\
\hline & Inter-observer & $3.97 \pm 4.05$ & -4.12 to 12.06 & 14.85 & $0.929(0.820,0.972)$ \\
\hline \multirow[t]{2}{*}{$L V E_{\|}$} & Intra-observer & $-0.22 \pm 0.89$ & -2.00 to 1.56 & 3.32 & $0.966(0.913,0.986)$ \\
\hline & Inter-observer & $-0.07 \pm 0.77$ & -1.61 to 1.47 & 2.88 & $0.969(0.921,0.988)$ \\
\hline \multirow[t]{2}{*}{$\mathrm{LV} E_{\mathrm{CC}}$} & Intra-observer & $-0.04 \pm 0.72$ & -1.48 to 1.41 & 2.15 & $0.990(0.975,0.996)$ \\
\hline & Inter-observer & $-0.44 \pm 1.31$ & -2.18 to 3.05 & 4.03 & $0.960(0.898,0.984)$ \\
\hline \multirow[t]{2}{*}{$L V E_{\mathrm{rr}}$} & Intra-observer & $-1.02 \pm 14.44$ & -29.89 to 27.85 & 11.28 & $0.793(0.477,0.918)$ \\
\hline & Inter-observer & $4.22 \pm 15.04$ & -25.85 to 34.29 & 12.54 & $0.832(0.576,0.934)$ \\
\hline \multirow[t]{2}{*}{$R \vee E_{\|}$} & Intra-observer & $-0.54 \pm 1.66$ & -3.86 to 2.78 & 3.92 & $0.975(0.937,0.990)$ \\
\hline & Inter-observer & $-4.43 \pm 3.18$ & -10.8 to 1.94 & 14.85 & $0.891(0.726,0.957)$ \\
\hline
\end{tabular}

$L A$, left atrium; $L V$, left ventricle; $R V$, right ventricle; $E_{\| l}$, longitudinal strain; $E_{\mathrm{cc}}$, circumferential strain; $E_{\mathrm{rr}}$, radial strain; ICC, intra-class coefficient; $C l$, confidence interval.

and longitudinal strains; in addition (iv) it provides quantitative ranges of LA, LV, and RV strain in healthy Chinese stratified into different age groups.

\section{Normal Ranges of Strain}

CMR-FT, a technique analogous to echocardiographic speckle tracking, has proven to be a feasible and reproducible approach for quantifying LA dynamics in terms of strain and strain rate (Kowallick et al., 2014, 2015b). Corresponding imaging biomarkers are increasingly recognized as having the potential to predict outcomes in a variety of cardiovascular disease states (Kowallick et al., 2014, 2015a; Inoue et al., 2015; Dick et al., 2017). The basic function reflected by LA strain depends on the use of either the QRS complex (R-R gating) or the $\mathrm{P}$ wave at the initiation of the calculation. When the $R$ wave is used, as in this study, the first peak between the $\mathrm{R}$ and $\mathrm{T}$ waves corresponds to reservoir function (Pathan et al., 2017). Reservoir strain of LA derived from CMR-FT in this study included $32.8 \pm 9.2 \%$ for longitudinal and $40.3 \pm 13.4 \%$ for circumferential strain. The longitudinal strain value was lower than $46 \pm 13 \%$ reported by Dick et al. (2017) from 25 healthy subjects. The lower LA volume in our subjects may explain the difference in $E_{11}$ values, since it has been reported that deformation parameters from CMR-FT for atrial reservoir functions are strongly related to volumetric indexes (Kowallick et al., 2014).

The LV myocardial $E_{11}$ and $E_{\mathrm{cc}}$ values obtained in our study were similar to the normal ranges of a recently published systematic review and meta-analysis (Vo et al., 2018). Andre et al. (2015) reported FT-derived LV longitudinal strain and circumferential strain values of $-21.6 \pm 3.2$ and $-21.3 \pm 3.3 \%$ in 150 healthy volunteers at $1.5 \mathrm{~T}$ (Achieva, Philips Medical Systems), which is concordant with our results. Taylor (Taylor et al., 2015), using CMR-FT at 1.5T (Magnetom Avanto, Siemens), reported values of $-21.3 \pm 4.8$ and $-26.1 \pm 3.8 \%$ derived from a group of 100 individuals comprising 10 men and women in each of 5 age deciles. The values are also in close agreement with our study. Relative greater radial strain exhibited in this study than previously results reported in (Andre et al., 2015; Taylor et al., 2015; Vo et al., 2018). It may be partly explained by that in our study, papillary muscles were included in the endocardial borders, which would have resulted in greater LV volume estimates with the consequent higher values for myocardial radial strain. Moreover, feature tracking imaging (FTI) algorithms inherently depend on image quality and endocardial border definition. And the large standard deviation of radial strain (up to $19.4 \%$ in this study) may suggest limitation for the present FTI algorithms in evaluating radial strain and advanced algorithms may be warranted. Our results showed regional variations that LV longitudinal and circumferential strain, and strain rate were highest in the lateral walls and lowest in the septum. Peak circumferential strain was lower in the mid-cavity than at the base or apex, which is consistent with results in Taylor et al. (2015).

The RV longitudinal strain measured in our study was consistent with that of Levy et al. (2014) who reported a range of -20.8 to $-34.1 \%$ (mean, $-29.0 \%$; $95 \%$ CI, -31.5 to $-26.5 \%$ ) based on a meta-analysis of two-dimensional speckle tracking echocardiographic-derived right ventricular strain in children. Our values for RV longitudinal strain were lower than those of Truong et al. (2017) and Liu et al. (2018), both post-processing with Circle Cardiovascular Imaging Tissue Tracking software. RV strains were obtained from 50 consecutive patients with no identified cardiac pathology $(-22.1 \pm 3.5 \%$; ages $4-81$ years; median age, 32 years) in Truong (Truong et al., 2017), and 100 healthy subjects containing 10 males and 10 females from each decade ( $-21.9 \pm 3.24 \%$; ages 20-70 years) (Liu et al., 2018). CMR-FT is based on the features at the myocardial boundary voxels and RV strain assessment software only measures endocardial strain, while Truong and Liu used both endocardial and epicardial borders to determine the myocardial deformable model. Secondly, RV strain assessment in our study included the septal values, as CMR-FT utilizes a LV tracking program, and Truong et al. (2017) only assessed the free wall without the inter-ventricular septum. More importantly, compared to CMR tissue tracking (CMR TT) based on the myocardium (Truong et al., 2017), manual intervention was needed to correct inaccurate tracking results in the feature tracking software, which could potentially introduce inconsistencies arising from image noise and the complex anatomical structure along the boundary. It would seem reasonable that differences in feature tracking 
software and strain assessment methods, a very thin free wall and presence of heavy trabeculations, variability among subjects, all combined to produce different values of RV longitudinal strain.

\section{Gender and Age Specific of Cardiac Deformation}

Conflicts remain regarding the effects of gender and age on cardiac deformation. Vo et al. (2018) showed no association of LV $E_{\mathrm{ll}}, E_{\mathrm{rr}}$ and RV $E_{\mathrm{ll}}$ with age, gender, software, field strength, sequence, LVEF or LV size. However, most published studies, such those as by Lawton et al. (2011), Augustine et al. (2013), and Taylor et al. (2015) reported greater strain in females, which is consistent with the trend in our results.

The LA $E_{11}, E_{\mathrm{cc}}$ and LV $E_{\mathrm{cc}}, E_{\mathrm{rr}}$ exhibited age dependency, although the correlation was weak. Systolic strain declined with increasing age. Higher correlation of LA strain with age compared to LV strain suggests greater clinical impact of age on LA strain. In contrast, age-related LV stiffness associated with a decline in diastolic function could be compensated for by increases in systolic wall thickening, thereby explaining the increase in radial strain with age. While our findings conflict with the result in Taylor et al. (2015), who reported an age-related increase in circumferential strain, our findings concur with those from Kuznetsova et al. (2008) and Dalen et al. (2010) who reported a decline in longitudinal strain associated with age. The discordance may be due to complexities in the course of aging rather than simply age and gender, hence the statistically significant but weak effects of age in our results.

Finally, we did not observe a significant association between age and RV longitudinal strain. This is consistent with the results from previous studies using speckle tracking echocardiography (Levy et al., 2014) and CMR tissue tracking (Truong et al., 2017; Liu et al., 2018; Vo et al., 2018).

\section{Reproducibility}

In this study, acceptable intra- and inter-observer agreement was found for peak systolic strain of LA, LV, and RV. Reproducibility obtained for LV $E_{\mathrm{cc}}$ was the best, followed by $E_{\mathrm{ll}}$ and then $E_{\mathrm{rr}}$. This is consistent with the findings of Taylor et al. (2015). It suggested that FTI allowed for reproducible quantification of systolic strains. And validation of LV $E_{\mathrm{rr}}$ is more challenging as this is generally less accurately quantified by all deformation algorithms.

\section{REFERENCES}

Andre, F., Steen, H., Matheis, P., Westkott, M., Breuninger, K., Sander, Y., et al. (2015). Age- and gender-related normal left ventricular deformation assessed by cardiovascular magnetic resonance feature tracking. J. Cardiovasc. Magn. Reson. 17:25. doi: 10.1186/s12968-015-0123-3

Augustine, D., Lewandowski, A. J., Lazdam, M., Rai, A., Francis, J., Myerson, S., et al. (2013). Global and regional left ventricular myocardial deformation measures by magnetic resonance feature tracking in healthy volunteers: comparison with tagging and relevance of gender. J. Cardiovasc. Magn. Reson. 15:8. doi: $10.1186 / 1532-429 X-15-8$

\section{Clinical Perspective}

CMR-FT derived wall motion assessment reliably quantifies LA, LV, and RV strain and SR from standard SSFP cine images. It seems a promising approach for the study of physiology in health and disease states. CMR-FT based global and segmental deformation quantification helps early detecting of subclinical myocardial dysfunction, monitoring the progress and predicting the outcome of the disease, such as heart failure with preserved ejection fraction (Zhong et al., 2013). Quantitative assessment of systolic right ventricular myocardial deformation can be used as a more quantitative tool to measure RV function in diseases such as repaired tetralogy of Fallot (Zhong et al., 2012) and pulmonary arterial hypertension (Leng et al., 2016).

\section{Limitations of Study}

Given the higher prevalence of risk factors such as diabetes mellitus, atherosclerosis, hypertension, and hyperlipidemia among the very elderly subjects, future studies may be required to focus on the normal ranges for these groups after stratification by risk factors. Other risk factors such as smoking, blood pressure, alcohol consumption are also missing in the present study, and how they will affect the strain values needs further investigation. Right atrial deformation was excluded from this analysis, as border tracking of the right atrium is known to be technically challenging owing to thin walls and the potential for morphological variations. This may be a contributing factor to the current lack of consensus on the clinical value of right atrial strain analysis.

\section{AUTHOR CONTRIBUTIONS}

$\mathrm{XM}, \mathrm{ML}$, and $\mathrm{LZ}$ conceived and designed the study. JP, XZ, LZ, ZF, ZW, HC, SL, R-ST, and AK performed the experiments. JP wrote the paper. XM, ML, LZ, ZF, XZ, and JA reviewed and edited the manuscript. All authors read and approved the manuscript.

\section{FUNDING}

This study was supported by the National Natural Science Foundation of China (81671647 and 81771787), the Beijing Natural Science Foundation Program (7172069), the China Postdoctoral Science Foundation (2016M592479), and the National Medical Research Council of Singapore (NMRC/TA/0031/2015 and NMRC/OFIRG/0018/2016).

Bellenger, N. G., Burgess, M. I., Ray, S. G., Lahiri, A., Coats, A. J., Cleland, J. G., et al. (2000). Comparison of left ventricular ejection fraction and volumes in heart failure by echocardiography, radionuclide ventriculography and cardiovascular magnetic resonance; are they interchangeable? Eur. Heart J. 21, 1387-1396. doi: 10.1053/euhj.2000.2011

Cerqueira, M. D., Weissman, N. J., Dilsizian, V., Jacobs, A. K., Kaul, S., Laskey, W. K., et al. (2002). Standardized myocardial segmentation and nomenclature for tomographic imaging of the heart. A statement for healthcare professionals from the Cardiac Imaging Committee of the Council on Clinical Cardiology of the American Heart Association. Circulation 105, 539-542. 
Dalen, H., Thorstensen, A., Aase, S. A., Ingul, C. B., Torp, H., Vatten, L. J., et al. (2010). Segmental and global longitudinal strain and strain rate based on echocardiography of 1266 healthy individuals: the HUNT study in Norway. Eur. J. Echocardiogr. 11, 176-183. doi: 10.1093/ejechocard/jep194

Dick, A., Schmidt, B., Michels, G., Bunck, A. C., Maintz, D., and Baeßler, B. (2017). Left and right atrial feature tracking in acute myocarditis: a feasibility study. Eur. J. Radiol. 89, 72-80. doi: 10.1016/j.ejrad.2017.01.028

Heermann, P., Hedderich, D. M., Paul, M., Schülke, C., Kroeger, J. R., Baeßler, B., et al. (2014). Biventricular myocardial strain analysis in patients with arrhythmogenic right ventricular cardiomyopathy (ARVC) using cardiovascular magnetic resonance feature tracking. J. Cardiovasc. Magn. Reson. 16:75. doi: 10.1186/s12968-014-0075-Z

Inoue, Y. Y., Alissa, A., Khurram, I. M., Fukumoto, K., Habibi, M., Venkatesh, B. A., et al. (2015). Quantitative tissue-tracking cardiac magnetic resonance (CMR) of left atrial deformation and the risk of stroke in patients with atrial fibrillation. J. Am. Heart Assoc. 4:e001844. doi: 10.1161/JAHA.115.001844

Kalam, K., Otahal, P., and Marwick, T. H. (2014). Prognostic implications of global LV dysfunction: a systematic review and meta-analysis of global longitudinal strain and ejection fraction. Heart 100, 1673-1680. doi: 10.1136/heartjnl-2014305538

Kawel-Boehm, N., Maceira, A., Valsangiacomo-Buechel, E. R., Vogel-Claussen, J., Turkbey, E. B., Williams, R., et al. (2015). Normal values for cardiovascular magnetic resonance in adults and children. J. Cardiovasc. Magn. Reson. 17:29. doi: 10.1186/s12968-015-0111-7

Khan, J. N., Singh, A., Nazir, S. A., Kanagala, P., Gershlick, A. H., and McCann, G. P. (2015). Comparison of cardiovascular magnetic resonance feature tracking and tagging for the assessment of left ventricular systolic strain in acute myocardial infarction. Eur. J. Radiol. 84, 840-848. doi: 10.1016/j.ejrad.2015. 02.002

Koh, A. S., Gao, F., Leng, S., Kovalik, J. P., Zhao, X. D., Tan, R. S., et al. (2018). Dissecting clinical and metabolomics associations of left atrial phasic function by cardiac magnetic resonance feature tracking. Sci. Rep. 8:8138. doi: 10.1038/ s41598-018-26456-8

Kowallick, J. T., Kutty, S., Edelmann, F., Chiribiri, A., Villa, A., Steinmetz, M., et al. (2014). Quantification of left atrial strain and strain rate using Cardiovascular Magnetic Resonance myocardial feature tracking: a feasibility study. J. Cardiovasc. Magn. Reson. 16:60. doi: 10.1186/s12968-0140060-6

Kowallick, J. T., Lotz, J., Hasenfuss, G., and Schuster, A. (2015a). Left atrial physiology and pathophysiology: role of deformation imaging. World J. Cardiol. 7, 299-305. doi: 10.4330/wjc.v7.i6.299

Kowallick, J. T., Morton, G., Lamata, P., Jogiya, R., Kutty, S., Hasenfuss, G., et al. (2015b). Quantification of atrial dynamics using cardiovascular magnetic resonance: inter-study reproducibility. J. Cardiovasc. Magn. Reson. 17:36. doi: 10.1186/s12968-015-0140-2

Kowallick, J. T., Morton, G., Lamata, P., Jogiya, R., Kutty, S., Lotz, J., et al. (2016). Inter-study reproducibility of left ventricular torsion and torsion rate quantification using MR myocardial feature tracking. J. Magn. Reson. Imaging 43, 128-137. doi: 10.1002/jmri.24979

Kramer, C. M., Barkhausen, J., Flamm, S. D., Kim, R. J., and Nagel, E. (2013). Society for cardiovascular magnetic resonance board of trustees task force on standardized protocols. Standardized cardiovascular magnetic resonance (CMR) protocols 2013 update. J. Cardiovasc. Magn. Reson. 15:91. doi: 10.1186/ 1532-429X-15-91

Kuznetsova, T., Herbots, L., Richart, T., D’Hooge, J., Thijs, L., Fagard, R. H., et al. (2008). Left ventricular strain and strain rate in a general population. Eur. Heart J. 29, 2014-2023. doi: 10.1093/eurheartj/ehn280

Lang, R. M., Bierig, M., Devereux, R. B., Flachskampf, F. A., Foster, E., Pellikka, P. A., et al. (2005). Recommendations for chamber quantification: a report from the American Society of Echocardiography's Guidelines and Standards Committee and the Chamber Quantification Writing Group, developed in conjunction with the European Association of Echocardiography, a branch of the European Society of Cardiology. J. Am. Soc. Echocardiogr. 18, 1440-1463. doi: 10.1016/j.echo.2005.10.005

Lawton, J. S., Cupps, B. P., Knutsen, A. K., Ma, N., Brady, B. D., Reynolds, L. M., et al. (2011). Magnetic resonance imaging detects significant sex differences in human myocardial strain. Biomed. Eng. Online 10:76. doi: 10.1186/1475-925X$10-76$
Leng, S., Zhao, X. D., Huang, F. Q., Wong, J. I., Su, B. Y., Allen, J. C., et al. (2015). Automated quantitative assessment of cardiovascular magnetic resonancederived atrioventricular junction velocities. Am. J. Physiol. Heart Physiol. 309, H1923-H1935. doi: 10.1152/ajpheart.0028.2015

Leng, S., Jiang, M., Zhao, X. D., Allen, J. C., Kassab, G., Ouyang, R. Z., et al. (2016). Three-dimensional tricuspid annular motion analysis from cardiac magnetic resonance feature-tracking. Ann. Biomed. Eng. 44, 3522-3538. doi: 10.1007/ s10439-016-1695-2

Leng, S., Zhang, S., Jiang, M., Zhao, X. D., Wu, R., Allen, J. C., et al. (2018). Imaging $4 \mathrm{D}$ morphology and dynamics of mitral annulus in humans using cardiac cine MR feature tracking. Sci. Rep. 8:81. doi: 10.1030/s41598-0171835-2

Levy, P. T., Sanchez, M. A., Machefsky, A., Fowler, S., Holland, M. R., and Singh, G. K. (2014). Normal ranges of right ventricular systolic and diastolic strain measures in children: a systematic review and meta-analysis. J. Am. Soc. Echocardiogr. 27, 549-560. doi: 10.1016/j.echo.2014.01.015

Liu, B., Dardeer, A. M., Moody, W. E., Edwards, N. C., Hudsmith, L. E., and Steeds, R. P. (2018). Normal values for myocardial deformation within the right heart measured by feature-tracking cardiovascular magnetic resonance imaging. Int. J. Cardiol. 252, 220-223. doi: 10.1016/j.ijcard.2017.10.106

Liu, H., Yang, D., Wan, K., Luo, Y., Sun, J. Y., Zhang, T. J., et al. (2017). Distribution pattern of left-ventricular myocardial strain analyzed by a cine MRI based deformation registration algorithm in healthy Chinese volunteers. Sci. Rep. 7:45314. doi: 10.1038/srep45314

Mangion, K., Clerfond, G., McComb, C., Carrick, D., Rauhalammi, S. M., McClure, J., et al. (2016). Myocardial strain in healthy adults across a broad age range as revealed by cardiac magnetic resonance imaging at 1.5 and 3.0T: associations of myocardial strain with myocardial region, age, and sex. J. Magn. Reson. Imaging 44, 1197-1205. doi: 10.1002/jmri.25280

Motoki, H., Borowski, A. G., Shrestha, K., Troughton, R. W., Tang, W. H., Thomas, J. D., et al. (2012). Incremental prognostic value of assessing left ventricular myocardial mechanics in patients with chronic systolic heart failure. J. Am. Coll. Cardiol. 60, 2074-2081. doi: 10.1016/j.jacc.2012. 07.047

Pathan, F., Elia, N. D., Nolan, M. T., Marwick, T. H., and Negishi, K. (2017). Normal ranges of left atrial strain by speckle-tracking echocardiography: a systematic review and meta-analysis. J. Am. Soc. Echocardiogr. 30, 59-70. doi: 10.1016/j.echo.2016.09.007

Prati, G., Vitrella, G., Allocca, G., Muser, D., Buttignoni, S. C., Piccoli, G., et al. (2015). Right ventricular strain and dyssynchrony assessment in arrhythmogenic right ventricular cardiomyopathy: cardiac magnetic resonance feature-tracking study. Circ. Cardiovasc. Imaging 8:e003647; discussione 003647. doi: 10.1161/CIRCIMAGING.115.003647

Rahman, Z. U., Sethi, P., Murtaza, G., Virk, H. U. H., Rai, A., Mahmod, M., et al. (2017). Feature tracking cardiac magnetic resonance imaging: a review of a novel non-invasive cardiac imaging technique. World J. Cardiol. 9, 312-319. doi: $10.4330 /$ wjc.v9.i4.312

Scatteia, A., Baritussio, A., and Bucciarelli-Ducci, C. (2017). Strain imaging using cardiac magnetic resonance. Heart Fail. Rev. 22, 465-476. doi: 10.1007/s10741017-9621-8

Stanton, T., Leano, R., and Marwick, T. H. (2009). Prediction of all-cause mortality from global longitudinal speckle strain: comparison with ejection fraction and wall motion scoring. Circ. Cardiovasc. Imaging 2, 356-364. doi: 10.1161/ CIRCIMAGING.109.862334

Taylor, R. J., Moody, W. E., Umar, F., Edwards, N. C., Taylor, T. J., and Stegemann, B. (2015). Myocardial strain measurement with feature-tracking cardiovascular magnetic resonance: normal values. Eur. Heart J. Cardiovasc. Imaging 16, 871-881. doi: 10.1093/ehjci/jev006

Truong, V. T., Safdar, K. S., Kalra, D. K., Gao, X., Ambach, S., Taylor, M. D., et al. (2017). Cardiac magnetic resonance tissue tracking in right ventricle: feasibility and normal values. Magn. Reson. Imaging 38, 189-195. doi: 10.1016/j.mri.2017. 01.007

Venkatesh, B. A., Donekal, S., Yoneyama, K., Wu, C., Fernandes, V. R., Rosen, B. D., et al. (2014). Regional myocardial functional patterns: quantitative tagged magnetic resonance imaging in an adult population free of cardiovascular risk factors: the multi-ethnic study of atherosclerosis (MESA). J. Magn. Reson. Imaging. 42, 153-159. doi: 10.1002/jmri. 24749 
Vo, H. Q., Marwick, T. H., and Negishi, K. (2018). MRI-derived myocardial strain measures in normal subjects. JACC Cardiovasc. Imaging 11, 196-205. doi: 10.1016/j.jcmg.2016.12.025

Zareian, M., Ciuffo, L., Habibi, M., Opdahl, A., Chamera, E. H., Wu, C. O., et al. (2015). Left atrial structure and functional quantitation using cardiovascular magnetic resonance and multimodality tissue tracking: validation and reproducibility assessment. J. Cardiovasc. Magn. Reson. 17:52. doi: 10.1186/ s12968-015-0152-y

Zhong, L., Gobeawan, L., Su, Y., Tan, J. L., Ghista, D. N., Chua, T., et al. (2012). Right ventricular regional wall curvedness and area strain in patients with repaired tetralogy of fallot. Am. J. Physiol. Heart. Circ. Physiol. 302, H1306H1316. doi: 10.1152/ajpheart.00679.2011

Zhong, L., Ng, K. K., Sim, L. L., Allen, J. C., Lau, Y. H., Sim, D. K., et al. (2013). Myocardial contractile dysfunction associated with increased 3-month and 1-year mortality in hospitalized patients with heart failure and preserved ejection fraction. Int. J. Cardiol. 168, 1975-1983. doi: 10.1016/j.ijcard.2012. 12.084

Zhang, L., Zhang, J., Han, W., Gao, J., He, L., Yang, Y., et al. (2016). Threedimensional rotation, twist and torsion analyses using real-time $3 \mathrm{~d}$ speckle tracking imaging: feasibility, reproducibility, and normal ranges in pediatric population. PLoS One 11:e0158679. doi: 10.1371/journal.pone.0158679

Zhao, X. D., Tan, R. S., Tang, H. C., Leng, S., Zhang, J. M., and Zhong, L. (2018). Analysis of three-dimensional endocardial and epicardial strains from cardiac magnetic resonance in healthy subjects and patients with hypertrophic cardiomyopathy. Med. Biol. Eng. Comput. 56, 159-172. doi: 10.1007/s11517017-1674-2

Conflict of Interest Statement: The authors declare that the research was conducted in the absence of any commercial or financial relationships that could be construed as a potential conflict of interest.

Copyright (c) 2018 Peng, Zhao, Zhao, Fan, Wang, Chen, Leng, Allen, Tan, Koh, $M a$, Lou and Zhong. This is an open-access article distributed under the terms of the Creative Commons Attribution License (CC BY). The use, distribution or reproduction in other forums is permitted, provided the original author(s) and the copyright owner(s) are credited and that the original publication in this journal is cited, in accordance with accepted academic practice. No use, distribution or reproduction is permitted which does not comply with these terms. 